\title{
Structural and electrical properties of swift heavy ion beam irradiated Co/Si interface
}

\author{
GARIMA AGARWAL, ANKUR JAIN, SHIVANI AGARWAL, D KABIRAJ ${ }^{\dagger}$ and I P JAIN* \\ Materials Science Laboratory, Centre for Non-Conventional Energy Resources, University of Rajasthan, \\ Jaipur 302 004, India \\ ${ }^{\dagger}$ Nuclear Science Centre, Aruna Asaf Ali Marg, New Delhi 110 067, India
}

MS received 8 August 2005; revised 2 February 2006

\begin{abstract}
Synthesis of swift heavy ion induced metal silicide is a new advancement in materials science research. We have investigated the mixing at $\mathrm{Co} / \mathrm{Si}$ interface by swift heavy ion beam induced irradiation in the electronic stopping power regime. Irradiations were undertaken at room temperature using $120 \mathrm{MeV}$ Au ions at the $\mathrm{Co} / \mathrm{Si}$ interface for investigation of ion beam mixing at various doses: $8 \times 10^{12}, 5 \times 10^{13}$ and $1 \times 10^{14} \mathrm{~cm}^{-2}$. Formation of different phases of cobalt silicide is identified by the grazing incidence $X$-ray diffraction (GIXRD) technique, which shows enhancement of intermixing and silicide formation as a result of irradiation. $I-V$ characteristics at $\mathrm{Co} / \mathrm{Si}$ interface were undertaken to understand the irradiation effect on conduction mechanism at the interface.
\end{abstract}

Keywords. Swift heavy ion; metal silicide; interface; GIXRD; I-V characteristics.

\section{Introduction}

Metal silicides are compounds, formed by metal on silicon exhibiting metallic conduction behaviour and have attracted world wide attention in the context of metallization applications because of their low metal like resistivity, high temperature stability and high electromigration resistance (Murarka 1983). Metal silicides are of particular interest, as their fabrication is compatible with silicon IC technologies (Yang et al 1995). Hence lot of work has been carried out to investigate different aspects of metal silicides produced by various means. Ion beam mixing is an advance technique for producing metal silicides, particularly for metastable phases and homogeneous mixing at the interface. Dufour (Dufour et al 1993) was first to observe the mixing in Fe/Si multilayer and found a quasi-complete mixing using $650 \mathrm{MeV} \mathrm{U}$ ions. Though a substantial amount of work has been done in ion beam induced mixing for the formation of metal silicides (Masoud et al 2002; Milosavljevic et al 2002; Dhar et al 2003), they are mostly in low energy region where elastic collisions (nuclear stopping) are predominant. Recently, researchers have shown interest (Assmann et al 1998; Kraft et al 2002; Chaudhary et al 2004; Diva et al 2004) in atomic mixing, materials modification and phase changes using ion beam mixing of high energy where inelastic collisions or electronic stopping are responsible for the energy loss. In the electronic slowing down regime, part of the energy of

\footnotetext{
*Author for correspondence (ipjain46@ sify.com)
}

incident ions is transferred to the host electrons, resulting in a high temperature increase for the electronic ionization (ionic spike), of $\sim 10^{-12} \mathrm{~s}$ duration (Wang et al 1994) and a high temperature increase of the electronic sub-system (thermal spike) causing a Gaussian like temperature profile around the centre of the ion track (Bolse and Schattat 2002). Depending on the energy deposited per unit length, the temperature in the core of the track may significantly rise above the melting point resulting in the formation of a molten cylinder of some $\mathrm{nm}$ in diameter. Within some tens of picoseconds this melt is then rapidly quenched to ambient temperature and a defect rich amorphous cylindrical zone is left behind. Hence ion-solid interaction is capable of producing electronic excitations leading to thermal spikes that produce defects along the path of ion resulting in mixing at the interface of metal silicon. It has already been suggested that several metals are sensitive to electronic energy loss $\left(S_{\mathrm{e}}\right)$ beyond certain threshold. The present study is undertaken for $S_{\mathrm{e}}$ sensitive, transitional metal $\mathrm{Co}$ for silicide formation and investigating its structural and electrical properties after SHI irradiation. Due to their good epitaxial and electrical properties (Kim et al 1997; Gambino and Colgan 1998), $\mathrm{CoSi}_{2}$ thin films are often employed in semiconductor technology and are used as buffer layer for the growth of new materials such as high temperature superconductor and are well suited for microelectronics because lattice mismatch between $\mathrm{CoSi}_{2}$ and $\mathrm{Si}$ is only $1 \cdot 2 \%$. The ion and energy for irradiation are chosen in such a way so as to produce the maximum possible electronic energy loss. 


\section{Experimental}

\subsection{Sample preparation}

Si (100) substrate was first chemically cleaned by a conventional procedure (trichloroethylene, acetone, methanol) and then dipped into a diluted HF solution before loading into vacuum evaporation chamber, to remove impurities or any oxide layer present. Thin Co metal film of $30 \mathrm{~nm}$ was deposited on $\mathrm{Si}(100)$ substrate using electron beam evaporation technique at NSC, Delhi, at room temperature (RT). Deposition was carried out under UHV conditions at a vacuum of $4 \times 10^{-8}$ Torr with a deposition rate of about $0 \cdot 1 \AA / s$. Thickness of the film was measured using Quartz Crystal Thickness Monitor.

\subsection{Irradiation}

For irradiation, the samples were mounted on a heavy copper ladder with good thermal contact using a thermally conducting adhesive ensuring negligible increase in sample temperature during irradiation. The $\mathrm{Co} / \mathrm{Si}$ samples were irradiated using $120 \mathrm{MeV}$ Au ions using 15 UD Pelletron Accelerator at NSC Delhi, at various fluences, $8 \times 10^{12}, 5 \times 10^{13}$ and $1 \times 10^{14}$ ions $/ \mathrm{cm}^{2}$ at RT. The irradiated fluences were estimated by measuring the ladder current under secondary electron suppressed geometry and considering the ion beam area. The samples were irradiated uniformly over an area of $1 \times 1 \mathrm{~cm}$ by scanning the ion beam of 1 pnA current using an electromagnetic scanner at $10^{-6}$ Torr vacuum.

Energy loss was estimated using simulation program TRIM (Zeigler et al 1985) and for $120 \mathrm{MeV} \mathrm{Au}$ in Co, the values of electronic energy and nuclear energy loss was $24.3 \mathrm{keV} / \mathrm{nm}$ and $0.44 \mathrm{keV} / \mathrm{nm}$, respectively, indicating that the dominant process of energy loss was due to elec- tronic excitation. Hence the observed mixing at the interface is due to the electronic energy deposition and the influence of nuclear energy loss is considered to be insignificant.

\subsection{GIXRD technique}

As deposited and irradiated samples were studied using GIXRD in IGCAR, Kalpakkam, to find out the presence of any phase or crystal structure at the interface. The difference between X-ray spectra of as deposited and irradiated samples allow to separate the pure irradiation effects created due to heavy ion irradiation. Diffraction measurements were performed using $\mathrm{CuK} \alpha(\lambda=1.54060 \AA)$ radiation. In order to limit the lateral divergence of the beam, slit of $0.05 \mathrm{~mm}$ was introduced in the incident path of rays before the sample.

\subsection{Electrical measurements}

The electrical measurements at $\mathrm{Co} / \mathrm{Si}$ interface were carried out by having a point contact on the front surface (irradiated side) and another contact on the back surface of the sample using silver paste. The current-voltage $(I-V)$ measurements at $\mathrm{Co} / \mathrm{Si}$ interface were carried out online in the Material Science Chamber at NSC, New Delhi, during the irradiation process.

\section{Results and discussion}

\subsection{Structural study by GIXRD}

Grazing incidence was used in order to prevent the radiation penetrating far into the substrate to hide the substrate peak of Si. Figure 1 shows diffraction spectrum of the as-

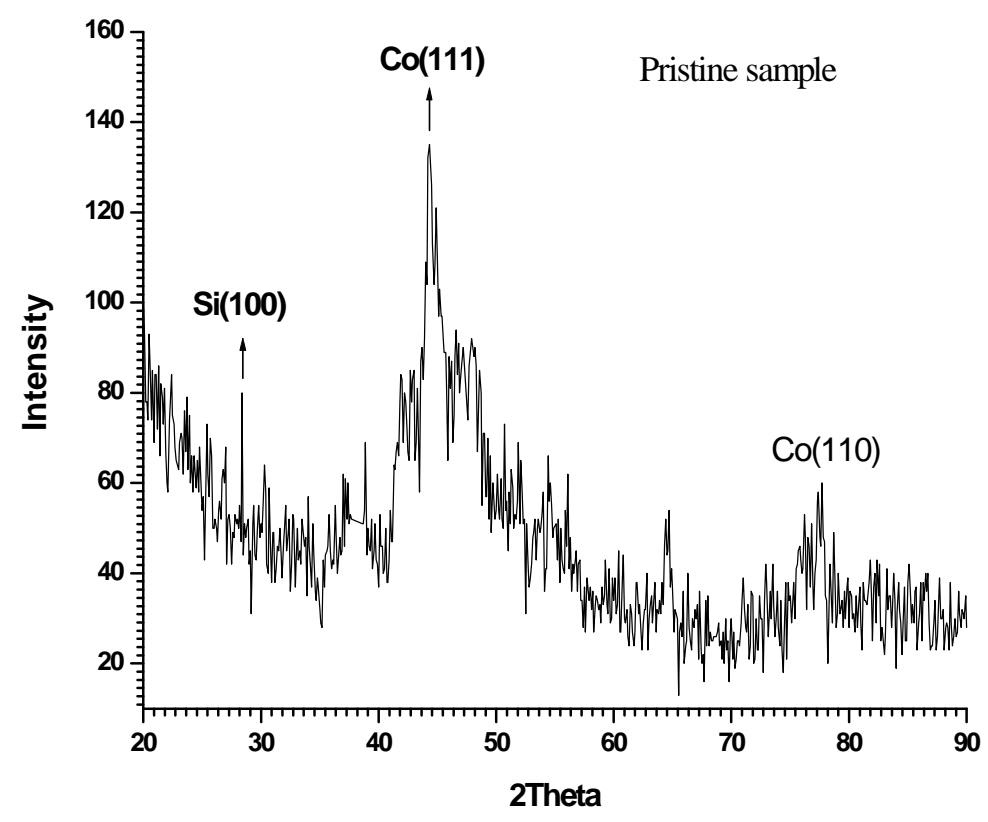

Figure 1. As deposited sample. 


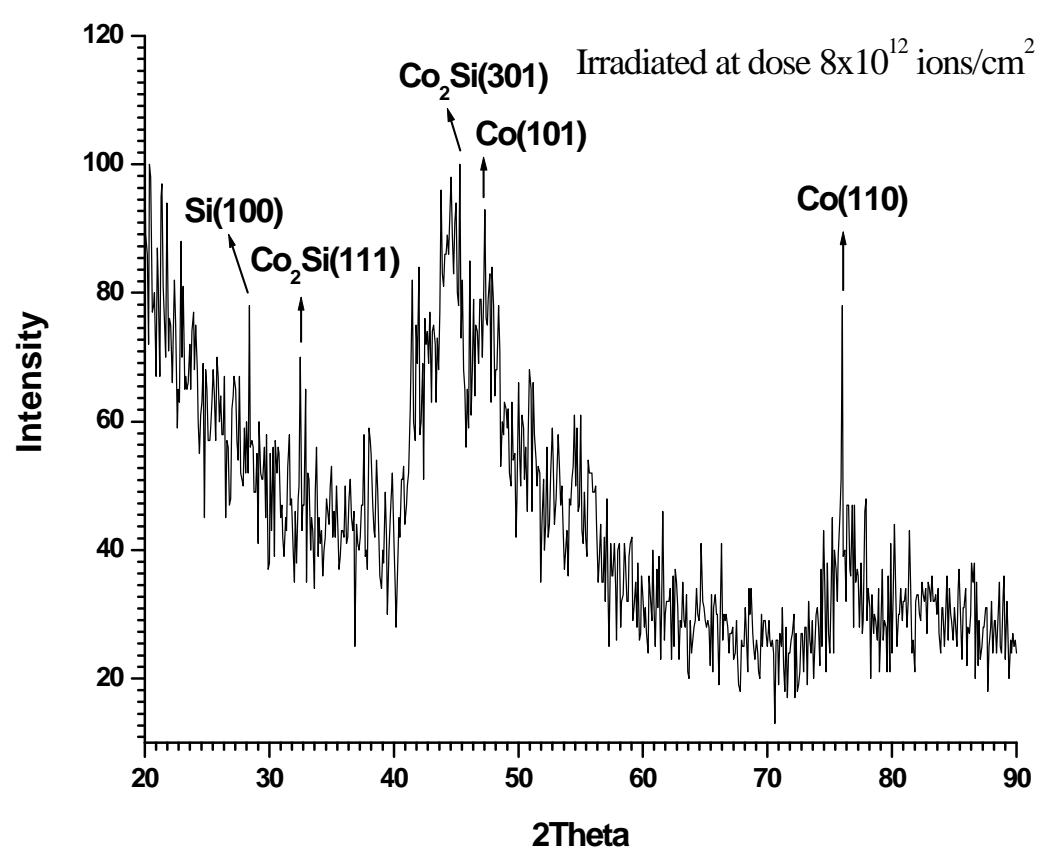

Figure 2. Irradiated sample with $120 \mathrm{MeV} \mathrm{Au}{ }^{+9}$ ions at a dose of $8 \times 10^{12}$ ions $/ \mathrm{cm}^{2}$.

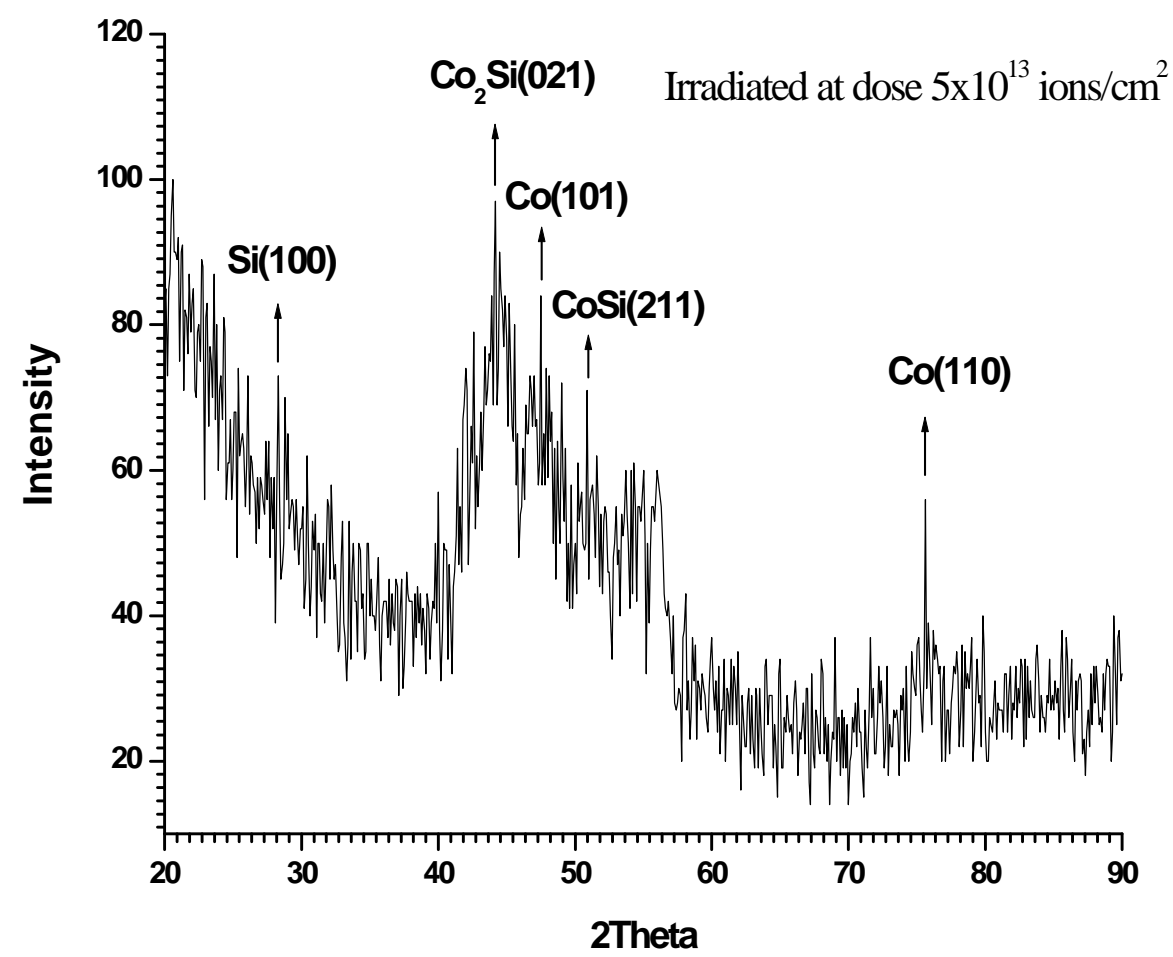

Figure 3. Irradiated sample with $120 \mathrm{MeV} \mathrm{Au}{ }^{+9}$ ions at a dose of $5 \times 10^{13}$ ions $/ \mathrm{cm}^{2}$.

deposited sample giving separate crystalline Bragg peaks corresponding to Co(111), Co (110) and $\mathrm{Si}(100)$, which does not show formation of any silicide. This system after irradiation at higher fluences shows crystalline peaks of
Co as well as Bragg peaks corresponding to different planes of $\mathrm{Co}_{2} \mathrm{Si}, \mathrm{CoSi}$ and $\mathrm{CoSi}_{2}$.

$\mathrm{XRD}$ measurements revealed that irradiation promotes the transformation of $\mathrm{Co}_{2} \mathrm{Si}$ to $\mathrm{CoSi}$ and $\mathrm{CoSi}$ to $\mathrm{CoSi}_{2}$. 
Figure 2 shows the pattern of irradiated $\mathrm{Co} / \mathrm{Si}$ sample at $8 \times 10^{12}$ ions $/ \mathrm{cm}^{2}$ fluence and it also shows the formation of crystalline $\mathrm{Co}_{2} \mathrm{Si}$ (111) and $\mathrm{Co}_{2} \mathrm{Si}$ (301) having a dominant peak of $\mathrm{Co}_{2} \mathrm{Si}$. Figure 3 shows GIXRD mea- surements of the same system irradiated at a fluence of $5 \times 10^{13}$ ions $/ \mathrm{cm}^{2}$ leading to the formation of $\mathrm{Co}_{2} \mathrm{Si}(021)$ and CoSi (211) indicating the formation of new phases on increasing the dose. In figure 4 the effect of irradiation

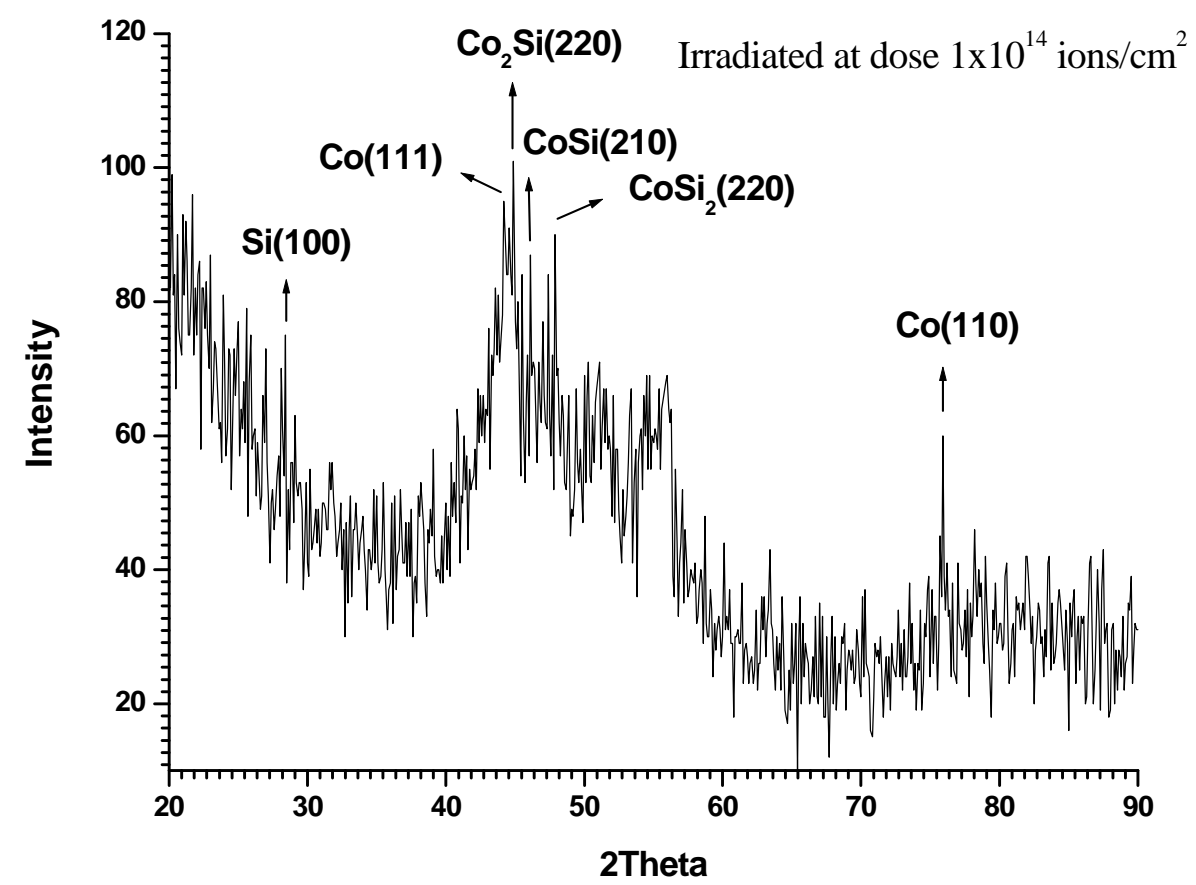

Figure 4. Irradiated sample with $120 \mathrm{MeV} \mathrm{Au}^{+9}$ ions at a dose of $1 \times 10^{14}$ ions $/ \mathrm{cm}^{2}$.

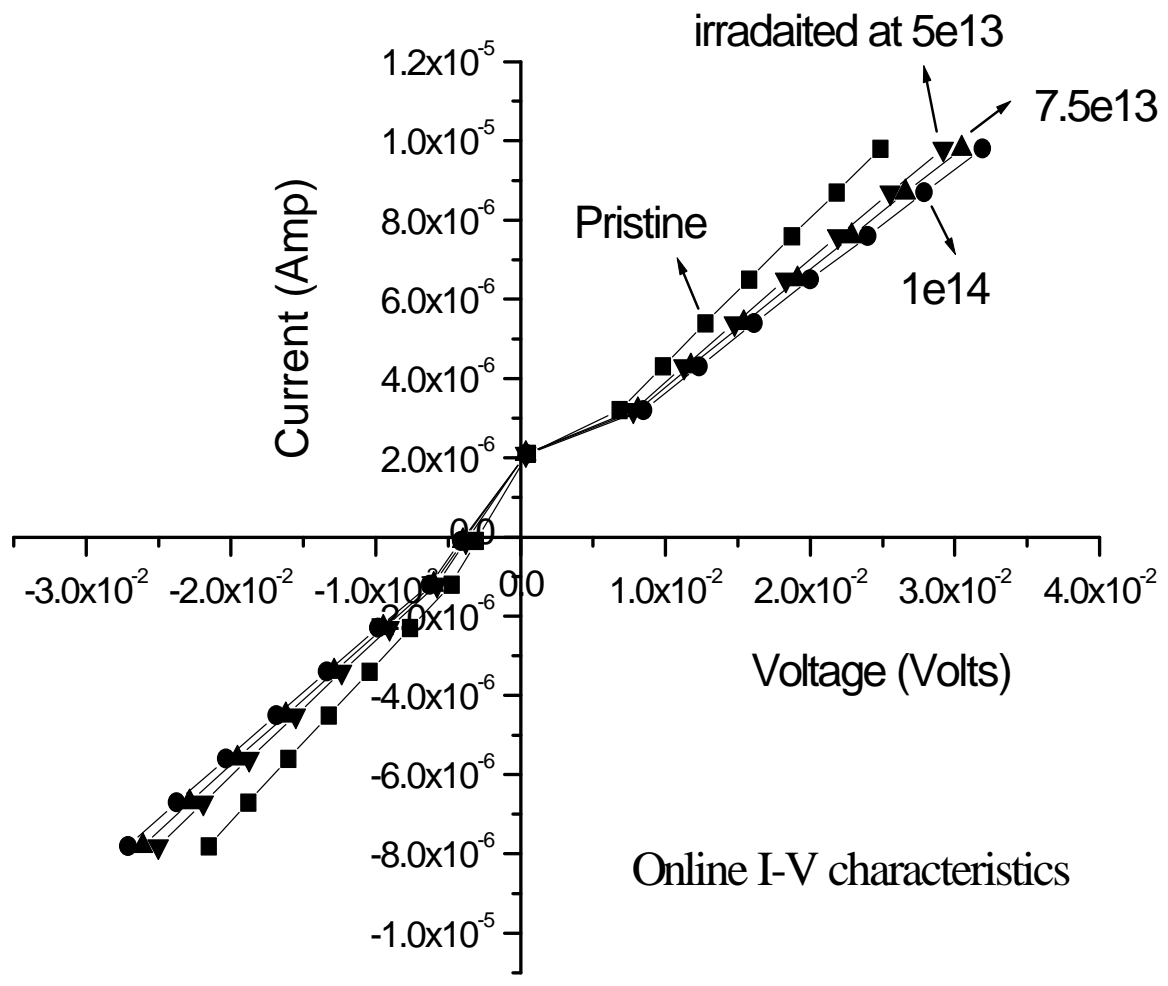

Figure 5. Online $I-V$ characteristics. 
using $1 \times 10^{14}$ ions $/ \mathrm{cm}^{2}$ at the interface shows the formation of three dominant peaks of three phases $\mathrm{Co}_{2} \mathrm{Si}$ (220), $\mathrm{CoSi}(210)$ and $\mathrm{CoSi}_{2}$ (220), where $\mathrm{CoSi}_{2}$ is the most stable phase.

All kinds of measurements show the Bragg peak of Si (100) and Co (111) at same intensity. These diffractograms and X-ray analysis clearly indicate the formation of different silicide phases at the interface. Hence ion-solid interaction is capable of producing electronic excitations leading to thermal spikes that produce defects along the path of ion resulting in mixing at the interface of metal silicon.

\subsection{Current-voltage characteristics}

$I-V$ characteristics have been measured for as deposited and samples irradiated at $5 \times 10^{13}, 7.5 \times 10^{13}$ and $1 \times 10^{14}$ ions $/ \mathrm{cm}^{2}$ at RT. It is seen from figure 5 that for all the samples the $I-V$ curves are linear. The effective resistance of the samples has been estimated from the linear portion of the $I-V$ curves. The room temperature resistance values were found to be $2.6 \mathrm{~K} \Omega, 3.4 \mathrm{~K} \Omega, 3.2 \mathrm{~K} \Omega$ and $3.1 \mathrm{~K} \Omega$ for the as deposited and irradiated at doses of $5 \times 10^{13}, 7 \cdot 5 \times 10^{13}$ and $1 \times 10^{14}$ ions $/ \mathrm{cm}^{2}$, respectively. It is clear from this data that for the sample irradiated at $5 \times 10^{13}$, the resistance value is greater than the asdeposited value. It is seen that the resistance of the interface decreases with increase in fluence. This higher value of resistance for lowest dose is due to generation of radiation induced defect states energy levels within the energy gap and compensate the free carriers in the substrate. Comparatively lower resistance values measured for samples irradiated at higher doses of $7.5 \times 10^{13}$ and $1 \times 10^{14}$ ions/ $\mathrm{cm}^{2}$ indicates conduction via the defect states and hence the electrical conduction in these samples is defect dominated. Therefore, we can go through least resistance values, which are lesser than the resistance value of as deposited, on increasing the fluence.

\section{Conclusions}

We have shown that electronic energy deposition in $\mathrm{Co} / \mathrm{Si}$ system results in strong interface mixing as soon as the electronic energy deposition exceeds a threshold value. GIXRD measurements clearly show that the mixing increases with increasing fluence and gives a stable structure at the highest fluence. $I-V$ curves show a linear behaviour for the as-deposited as well as for irradiated samples indicating an ohmic contact at the interface. Since all the irradiated samples have more resistance value than as- deposited, we therefore, conclude that due to irradiation the resistance first increases due to creation of amorphous state, which converts to crystalline on irradiation at higher doses resulting in the decrease in resistance. Hence SHI induced ion beam mixing (IBM) is a powerful tool for the synthesis of silicides in various compound phases and it is perhaps the best way to get the stable and homogeneous phases of metal silicides.

\section{Acknowledgements}

The authors gratefully acknowledge Mrs Kalawati and Dr V S Shastri, Materials Science Division, Indira Gandhi Centre for Atomic Research, Kalpakkam, for providing grazing incidence X-ray diffractogram. One of the authors (GA) is thankful to NSC, New Delhi, for providing a fellowship. The work was carried out under the financial support from NSC, New Delhi.

\section{References}

Assmann W, Dobler M, Avasthi D K, Kruijer S, Mieskes H D and Nolte H 1998 Nucl. Instrum. Meth. Phys. Res. B146 271

Bolse W and Schattat B 2002 Nucl. Instrum. Meth. Phys. Res. B190 173

Chaudhary S, Biswas S, Gupta A, Avasthi D K, Bhattacharyya D, Teichert S and Sarkar D K 2004 Nucl. Instrum. Meth. Phys. Res. B217 589

Dhar S, Schaaf P, Lieb K P, Bibic N, Milosavljevic M, Sajavaara T, Keinonen J and Traverse A 2003 Nucl. Instrum. Meth. Phys. Res. B205 741

Diva K, Kabiraj D, Chakraborty B R, Shivaprasad S M and Avasthi D K 2004 Nucl. Instrum. Meth. Phys. Res. B222 169

Dufour C, Bauer Ph, Marchal G and Jousset J C 1993 Europhysics Lett. 21671

Gambino J P and Colgan E G 1998 Mater. Chem. Phys. 5299

Kim G B, Kwak J S and Baik H K 1997 J. Appl. Phys. 82 2323

Kraft S, Schattat B and Bolse W 2002 J. Appl. Phys. 911129

Masoud N M, Arafah D E and Beeker K H 2002 Nucl. Instrum. Meth. Phys. Res. B198 64

Milosavljevic M, Shao G, Bibic N, McKinty C N, Javnes C and Homewood K P 2002 Nucl. Instrum. Meth. Phys. Res. $\mathbf{B 1 8 8}$ 166

Murarka S P 1983 Silicides for VLSI applications (New York: Academic Press)

Wang Z G, Dufour C, Paumier E and Toulemonde M 1994 J. Phys. Condens. Matter 66733

Yang Z, Homewood K P, Finney M S, Harry M A and Reeson K J 1995 J. Appl. Phys. 781958

Zeigler J F, Biersack J P and Littmark U 1985 The stopping and ranges of ions in solids (New York: Pergamon) 